\title{
Average refractive index of tendon as a function of water content
}

\author{
Marina E. Shvachkina*, Dmitry D. Yakovlev, Alexander B. Pravdin, and Dmitry A. Yakovlev \\ Department of Optics and Biophotonics, Faculty of Physics, Saratov State University, 83 Astrakhanskaya st, \\ Saratov 410012, Russia \\ *e-mail: marevesh@mail.ru
}

\begin{abstract}
Experimental data on the dependence of the average refractive index of rat tail tendon (RTT) on water content are reported. Using optical coherence tomography, the average group refractive index (at a wavelength of $930 \mathrm{~nm}$ ) and cross-section area of rat tail tendon fascicle specimens during their air-drying and rehydration were monitored. The dependence of the average group refractive index of RTT $\left(n_{g}\right)$ on the volume fraction of water $\left(C_{w}\right)$ has been found to be nonlinear and to be well approximated by the quadratic polynomial $n_{g}=1.5713-0.1969 C_{w}-0.0328\left(C_{w}\right)^{2}$. The reported data are shown to be in good agreement with previously published data for bovine cornea. (C) 2018 Journal of Biomedical Photonics \& Engineering.
\end{abstract}

Keywords: collagen; tissue; refractive index; water content; hydration.

Paper \#3268 received 23 Dec 2017; revised manuscript received 1 Mar 2018; accepted for publication 16 Mar 2015; published online 31 Mar 2018. doi: 10.18287/JBPE18.04.010302.

\section{References}

1. S. L. Jacques, "Optical properties of biological tissues: a review," Physics in Medicine and Biology 58(11), R37-R61 (2013).

2. F. P. Bolin, L. E. Preuss, R. C. Taylor, and R. J. Ference, "Refractive index of some mammalian tissues using a fiber optic cladding method," Applied Optics 28(12), 2297-2303 (1989).

3. G. J. Tearney, M. E. Brezinski, B. E. Bouma, M. R. Hee, J. F. Southern, and J. G. Fujimoto, "Determination of the refractive index of highly scattering human tissue by optical coherence tomography," Optics Letters 20(21), 2258-2260 (1995).

4. A. R. Knuettel and M. Boehlau-Godau, "Spatially confined and temporally resolved refractive index and scattering evaluation in human skin performed with optical coherence tomography," Journal of Biomedical Optics 5(1), 83-93 (2000).

5. M. Ohmi, Y. Ohnishi, K. Yoden, and M. Haruna, "In vitro simultaneous measurement of refractive index and thickness of biological tissue by the low coherence interferometry," IEEE Transactions on Biomedical Engineering 47(9), 1266-1270 (2000).

6. X. Wang, C. Zhang, L. Zhang, L. Xue, and J. Tian, "Simultaneous refractive index and thickness measurements of bio-tissue by optical coherence tomography," Journal of Biomedical Optics 7(4), 628-632. (2002).

7. Y. L. Kim, J. T. Walsh Jr, T. K. Goldstick, and M. R. Glucksberg, "Variation of corneal refractive index with hydration," Physics in Medicine and Biology 49(5), 859-868 (2004).

8. J. Lai, Z. Li, C. Wang, and A. He, "Effective refractive indices of biological tissues and its experimental determination," Proceedings of SPIE 5630 (2005).

9. H. Ding, J. Q. Lu, W. A. Wooden, P. J. Kragel, and X. H. Hu, "Refractive indices of human skin tissues at eight wavelengths and estimated dispersion relations between 300 and $1600 \mathrm{~nm}$," Physics in Medicine and Biology 51(6), 1479-1489 (2006).

10. J. Kim, D. P. Dave, C. G. Rylander, J. Oh, and T. E. Milner, "Spatial refractive index measurement of porcine artery using differential phase optical coherence microscopy," Lasers in Surgery and Medicine 38(10), 955959 (2006).

11. J. Sun, S. J. Lee, L. Wu, M. Sarntinoranont, and H. Xie, "Refractive index measurement of acute rat brain tissue slices using optical coherence tomography," Optics Express 20(2), 1084-1095 (2012). 
12. D. Maciel, S. P. Veres, H. J. Kreuzer, and L. Kreplak, "Quantitative phase measurements of tendon collagen fibres," Journal of Biophotonics 10(1), 111-117 (2017).

13. Z. C. Deng, J. Wang, Z. X. Hu, J. C. Mei, and Q. Ye, "Study on the relation between the refractive index of fresh muscle tissue and its water content from 400 to $750 \mathrm{~nm}$," Journal of Modern Optics 65(4), 451-455 (2017).

14. A. M. Zysk, S. G. Adie, J. J. Armstrong, M. S. Leigh, A. Paduch, D. D. Sampson, F. T. Nguyen, and S. A. Boppart, "Needle-based refractive index measurement using low-coherence interferometry," Optics Letters 32(4), 385-387 (2007).

15. L. Oliveira, A. Lage, M. P. Clemente, and V. V. Tuchin, "Optical characterization and composition of abdominal wall muscle from rat," Optics and Lasers in Engineering 47(6), 667-672 (2009).

16. T. K. Biswas and T. M. Luu, "In vivo MR measurement of refractive index, relative water content and T2 relaxation time of various brain lesions with clinical application to discriminate brain lesions," Internet Journal of Radiology 13(1), 1 (2011).

17. P. Fratzl, “Collagen: structure and mechanics, an Introduction," in Collagen, Springer, New York, USA, 1-13 (2008).

18. P. Kannus, "Structure of the tendon connective tissue," Scandinavian journal of medicine and science in sports 10(6), 312-320 (2000).

19. H. R. Screen, V. H. Chhaya, S. E. Greenwald, D. L. Bader, D. A. Lee, and J. C. Shelton, "The influence of swelling and matrix degradation on the microstructural integrity of tendon," Acta Biomaterialia 2(5), 505-513 (2006).

20. A. A. de Aro, B. de Campos Vidal, and E. R. Pimentel, "Biochemical and anisotropical properties of tendons," Micron 43(2), 205-214 (2012).

21. J. Kastelic, A. Galeski, and E. Baer, "The multicomposite structure of tendon," Connective Tissue Research 6(1), 11-23 (1978).

22. R. W. D. Rowe, "The structure of rat tail tendon," Connective Tissue Research 14(1), 9-20 (1985).

23. V. V. Tuchin, Tissue optics: light scattering methods and instruments for medical diagnosis, SPIE press, Bellingham, Washington (2015).

24. A. T. Yeh, B. Choi, J. S. Nelson, and B. J. Tromberg, "Reversible dissociation of collagen in tissues," Journal of Investigative Dermatology 121(6), 1332-1335 (2003).

25. W. V. Sorin, and D. F. Gray, "Simultaneous thickness and group index measurement using optical lowcoherence reflectometry," IEEE Photonics Technology Letters 4(1), 105-107 (1992).

26. X. J. Wang, T. E. Milner, M. C. Chang, and J. S. Nelson, "Group refractive index measurement of dry and hydrated type I collagen films using optical low-coherence reflectometry," Journal of Biomedical Optics 1(2), 212-217 (1996).

27. Z. Bor, K. Osvay, B. Racz, and G. Szabo, "Group refractive index measurement by Michelson interferometer," Optics Communications 78(2), 109-112 (1990).

28. M. Born, and E. Wolf, Principles of optics: electromagnetic theory of propagation, interference and diffraction of light, Cambridge University Press, Cambridge (1999).

29. D. J. Segelstein, The complex refractive index of water, Doctoral dissertation, University of Missouri-Kansas City, Kansas City (1981).

30. F. De Chaumont, S. Dallongeville, N. Chenouard, N. Herve, S. Pop, T. Provoost, V. Meas-Yedid, P. Pankajakshan, T. Lecomte, Y. Le Montagner, T. Lagache, A.Dufour, and J.-C. Olivo-Marin, "Icy: an open bioimage informatics platform for extended reproducible research," Nature Methods 9(7), 690-696 (2012).

31. D. M. Maurice, "The structure and transparency of the cornea," The Journal of Physiology 136(2), 263-286 (1957).

32. K. M. Meek, N. J. Fullwood, P. H. Cooke, G. F. Elliott, D. M. Maurice, A. J. Quantock, R. S. Wall, and C. R. Worthington, "Synchrotron x-ray diffraction studies of the cornea, with implications for stromal hydration," Biophysical Journal 60(2), 467-474 (1991).

33. E. P. Katz, and S. T. Li, "Structure and function of bone collagen fibrils," Journal of Molecular Biology 80(1), $1-15$ (1973).

34. D. W. Leonard, and K. M. Meek, "Refractive indices of the collagen fibrils and extrafibrillar material of the corneal stroma,” Biophysical Journal 72(3), 1382-1387 (1997).

35. C. Morin, C. Hellmich, and P. Henits, "Fibrillar structure and elasticity of hydrating collagen: a quantitative multiscale approach,” Journal of Theoretical Biology 317, 384-393 (2013).

36. S. Hayes, T. White, C. Boote, C. S. Kamma-Lorger, J. Bell, T. Sorenson, N. Terrill, O. Shebanova, and K. M. Meek, "The structural response of the cornea to changes in stromal hydration," Journal of The Royal Society Interface 14(131), 20170062 (2017).

37. J. C. Martinez-Anton, and E. Bernabeu, "Spectrogoniometry and the WANTED method for thickness and refractive index determination," Thin Solid Films 313, 85-89 (1998). 


\section{Introduction}

One of the optical parameters that can be directly measured for biological tissues is the average refractive index of the tissue [1-15]. In the literature, one can find estimates of the average refractive index for many types of tissues (cornea, sclera, dermis, epidermis, brain tissue etc.; see, e.g., [1, 2, 4, 7-13, 15, 16]). The average refractive index depends on tissue composition and, in particular, on the water content [1]. The water content is one of the most variable and important tissue parameters, and the possibility to evaluate it from the measured values of the average tissue refractive index is of great practical interest. However, to date direct measurements of the average tissue refractive index as a function of tissue water content have been made only for a small number of tissues, e.g., muscle tissue [13, 15] and brain tissues [1, 16]. Of collagenous tissues, as far as we are aware, reliable estimates of the average refractive index as a function of water content were obtained only for cornea [7], which is a tissue with a very high water content - normal cornea contains about $76 \mathrm{wt} \%$ water and $15 \mathrm{wt} \%$ collagen [17]. In this paper we present experimental estimates for the average tissue refractive index as a function of water content for tendon, a collagenous tissue with the highest normal collagen content-tendons contain typically 24-38 wt \% collagen and 55-72 wt \% water [18-20]. The experiments reported here were performed on rat tail tendon (RTT) fascicles. Fascicles are secondary collagen bundles of tendons [20-22]. Their composition and collagen fibril packing are similar to those of collagen fibers of dermis and sclera [17]. But fascicles, whose diameter typically lies within the range from 250 to $500 \mu \mathrm{m}$, are tens times thicker than dermal and scleral collagen fibers, which makes them quite convenient for experimentation. Due to their relatively simple structure, ease of extraction and manipulation, and ready availability, RTT fascicles are a very popular model object for studying physical and physiological properties of collagen fibers and collagenous tissues. For example, they are used in studying the effect of immersion agents, which are employed in the immersion optical clearing technique [23], on collagen bundles (see, e.g., [24]). The quantitative data presented here can be used in such studies as reference data. For example, these data can be used for evaluating the duration of the solely dehydration stage of the immersion agent - biotissue interaction.

\section{Methods}

In order to determine the average refractive index of RTT fascicles as a function of water content, we monitored, using optical coherence tomography (OCT), the cross-section area and average refractive index of RTT fascicle specimens during their air-drying from the native state to the air-dry state and in the process of rehydration of dried specimens in normal saline solution (NSS: aqueous solution of $0.9 \mathrm{wt} \% \mathrm{NaCl}$ ). A series of measurements began with the measurement of the cross- section area and refractive index of the sample in its initial (native) state. The fascicle, being submerged in NSS, was mounted, in a slightly stretched state, on an object-plate using binder clips and covered with a cover-slip. Then it was placed in the OCT beam path so that the direction of the fascicle was perpendicular to the B-scan direction in order to obtain an OCT-scan of the cross-section of the fascicle (Fig. 1a). After that, the cover-slip was removed, and the NSS was quickly removed from the surface of the object-plate with a filter paper. The (non-covered) sample was placed on the OCT scanner stage in the same position as before in order to monitor the sample parameters during its dehydration in air at room temperature (Fig. 1b, c). To estimate the parameters of the sample in the standard air-dry state, the sample was allowed to dry for $1 \mathrm{hr}$ at $105^{\circ} \mathrm{C}$ (Fig. 1d). For rehydration measurements, the dried fascicle on the object-plate was surrounded by a large amount of NSS and covered by a cover-slip.

One of the parameters that was traced in the experiment was the coefficient of fascicle volume change (volume factor)

$$
k_{s}=V / V_{0}
$$

where $V$ is the current value of tissue volume and $V_{0}$ is the volume of the tissue in the initial state. In our experiments, the tendon fascicle with fixed ends remained slightly stretched during both the dehydration and following rehydration, i.e. the length of the fascicle did not change. Therefore the volume factor can be calculated as

$$
k_{s}=S / S_{0}
$$

where $S$ and $S_{0}$ are the values of the cross-section area at the moment of measuring and in the initial state, respectively.

The measured values of $k_{s}$ were used for estimating the water volume fraction $C_{w}$ in the tissue. By definition,

$$
C_{w}=V_{w} / V
$$

where $V$ is the volume of the tissue and $V_{w}$ is the volume of water in the tissue. On the assumption that to a good accuracy

$$
V=V_{d r y}+V_{w}
$$

where $V_{d r y}$ is the volume of the tissue in the dry state, it follows from (1) and (3) that

$$
C_{w} \approx \frac{k_{s}-k_{s d r y}}{k_{s}}
$$




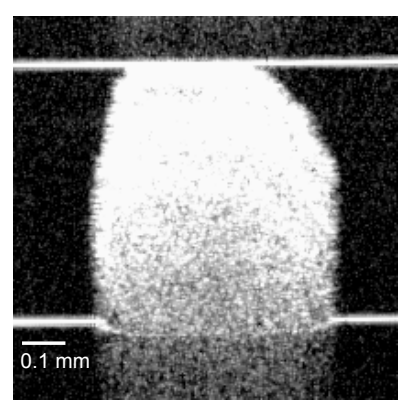

(a)

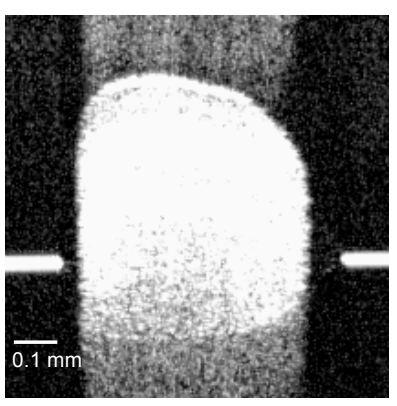

(b)

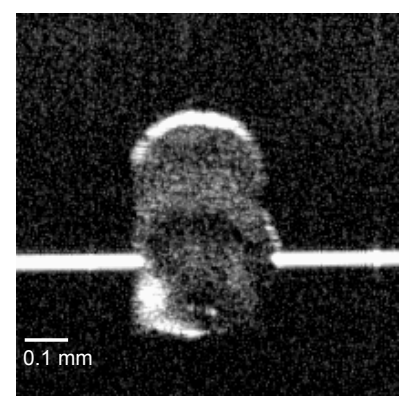

(c)

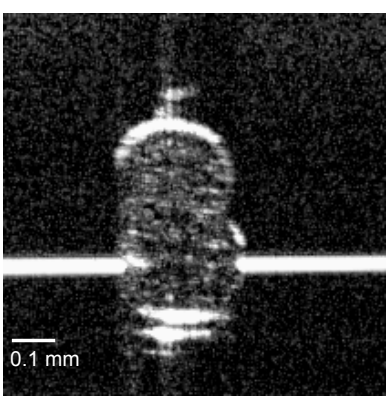

(d)

Fig. 1 OCT-images of a transverse cross-section of an RTT fascicle (a) in normal saline solution (native state), (b) after air-drying at room temperature for $1.7 \mathrm{~min}$, (c) after air-drying at room temperature for 24 min, and (d) after air-drying at $105^{\circ} \mathrm{C}$ for 1 hour.

where $k_{s d r y}$ is the value of $k_{s}$ for the dry state. For the native state, $C_{w}=1-k_{s d r y}$.

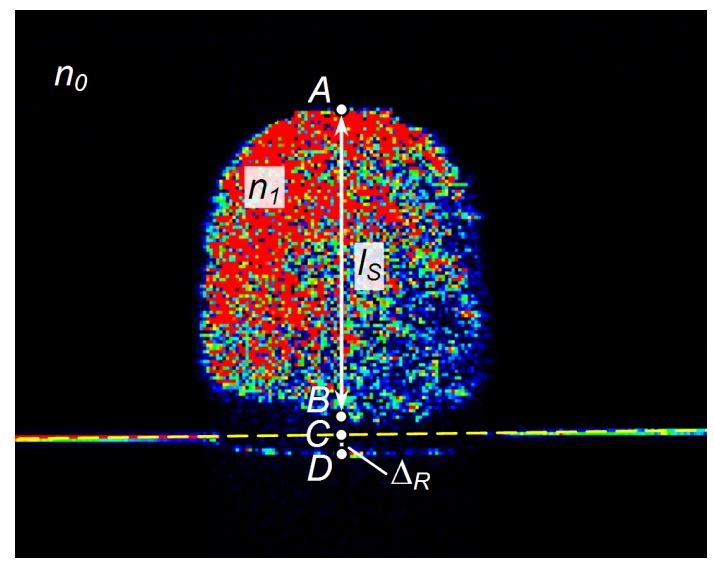

Fig. 2 Parameters used in the calculations of the average group refractive index of the tissue from an OCT-image of the specimen.

To estimate the average refractive index of the tissue using OCT, we employed a common method [3, 7, 25, $26]$ in which the group refractive index and physical thickness of the sample are determined from the measured values of the optical path length $l_{S}=n_{1} d$, where $d$ is the physical thickness of the sample for the given A-scan line and $n_{1}$ is the average group refractive index of the sample for this scan line, and shift $\Delta_{R}$ of the image of a reflecting surface arranged beyond the sample with respect to its position in the absence of the sample (in our experimental geometry, as in [26], the role of this reflecting surface is played by the frontal surface of the object-plate (glass substrate); see Fig.2). In an example in Fig. 2, where an OCT-image of a transverse section of an RTT fascicle is shown, apart from a common scale factor, $l_{S}=|\mathrm{AB}|$ and $\Delta_{R}=|\mathrm{CD}|$. Given $l_{S}$ and $\Delta_{R}$, the average refractive index of the sample $n_{1}$ for the A-scan line under consideration can be calculated as

$$
n_{1}=\frac{n_{0} l_{S}}{l_{S}-\Delta_{R}}
$$

where $n_{0}$ is the group refractive index of the surrounding medium (in our experiments, air or NSS). We calculated the average group refractive index of the specimen for the given cross-section as an average over several (3 to 5) A-scan lines. The group refractive index of the surrounding medium was calculated from dispersion data for the phase refractive index of the medium using the following common relation $[27,28]$ :

$$
n_{g}\left(\lambda_{0}\right)=n_{p}\left(\lambda_{0}\right)-\left.\lambda_{0} \frac{d n_{p}}{d \lambda}\right|_{\lambda=\lambda_{0}}
$$

where $n_{p}$ is the phase refractive index, $n_{g}$ is the group refractive index, and $\lambda_{0}$ is the central wavelength of the probing radiation in vacuum. For the OCT-system that we used, ThorLabs-OCP930SR, $\lambda_{0}=930 \mathrm{~nm}$. We took $n_{g}(930 \mathrm{~nm})=1$ for air and $n_{g}(930 \mathrm{~nm})=1.3416$ for NSS. The phase refractive index of NSS was assumed to be approximately equal to the phase refractive index of water. For water, we used the dispersion data reported in [29], which gave the mentioned value of 1.3416 .

The area $S$ [see(2)] was calculated using the formula

$$
S=\frac{S_{\text {OCT-pix }} k_{p m^{2}}}{n_{g t}},
$$

where $S_{\text {OCT-pix }}$ is the area of the specimen cross-section in the OCT-image in pixels, $k_{p m^{2}}$ is an instrument scale factor (in our case, $k_{p m^{2}}=1.2632 \cdot 10^{-5} \mathrm{~mm}^{2} / \mathrm{pixel}$ ), and $n_{g t}$ is the value of the average group refractive index of the specimen calculated from the OCT data for this cross-section as described above. To find $S_{\text {OCT-pix }}$ we used the open-source image analysis software Icy [30].

An example of the measured time-dependences of the volume factor $k_{s}(2)$ and the average group refractive index of the tissue in the process of air-drying for a sample of RTT fascicle is presented in Fig. 3. It is clearly seen that the shrinkage of the tissue due to water 
loss is accompanied by an increase in its average refractive index.

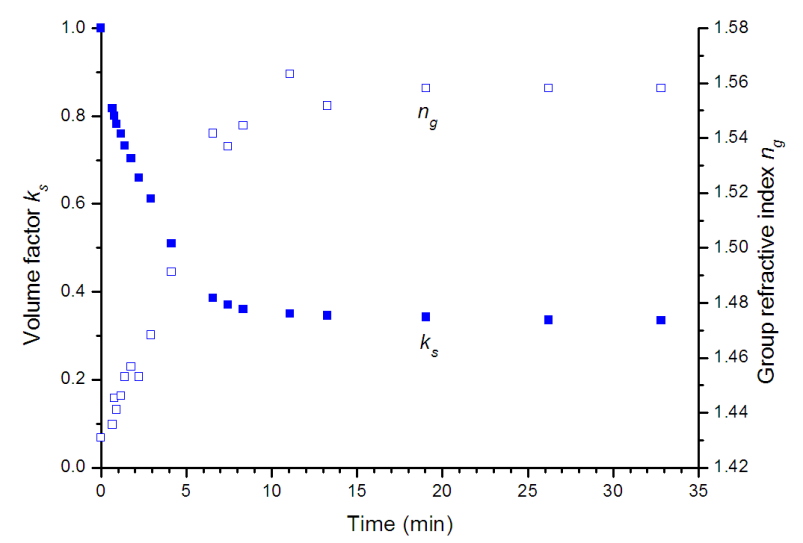

Fig. 3 Change of the volume factor $k_{s}$ and average refractive index $n_{g}$ of an RTT fascicle specimen during air-drying at room temperature.

Since the samples were relatively small in size, the boundaries of the sample and the upper boundary of the object-plate under the sample were clearly visible in OCT images even when the sample was in the native state, and their position could be determined with good accuracy. This ensured high accuracy of cross-section area measurements, which is confirmed by a small scatter of points in $k_{s} v s$ time plots (as an example see Fig. 3). At the same time the samples were sufficiently thick for the relative accuracy of group refractive index measurements to be of the order of $0.3-0.5 \%$.

\section{Samples}

Fascicles were excised from the tails of mature rats within one hour after decapitation, then immediately immersed in NSS. All refractive-index measurements were performed on fascicles ranging in diameter from 300 to $400 \mu \mathrm{m}$. The storage time of samples in NSS before they were used in experiments did not exceed 7 days. No statistically significant change (Friedman test, probability value $p=0.32$ ) in the average refractive index of RTT fascicle specimens was observed during 7-day storage in NSS (see Fig. 4).

The studies were approved by the Ethics Committee of Saratov State Medical University (Saratov, Russia).

\section{Results and discussion}

Fig. 5 shows the measured dependences of the average refractive index, $n_{g}$, on the volume factor $k_{s}$ for four samples of RTT fascicles, samples $1,2,3$, and 4 . The OCT data for sample 1 were collected during $30 \mathrm{~min}$ when this sample was dried at room temperature. Sample 2 was dried at room temperature for $2 \mathrm{hr}$, then held for $1 \mathrm{hr}$ at $50^{\circ} \mathrm{C}$, and finally held for $1 \mathrm{hr}$ at $105^{\circ} \mathrm{C}$. Sample 3 was dried for $40 \mathrm{~min}$ at room temperature and then for $1 \mathrm{hr}$ at $105^{\circ} \mathrm{C}$. Sample 4 was first dried at room temperature for $20 \mathrm{~min}$ and then placed in NSS for rehydration. From Fig. 5, it can be seen that the data for different samples are in good agreement, as are the data for drying and rehydration of sample 4 .

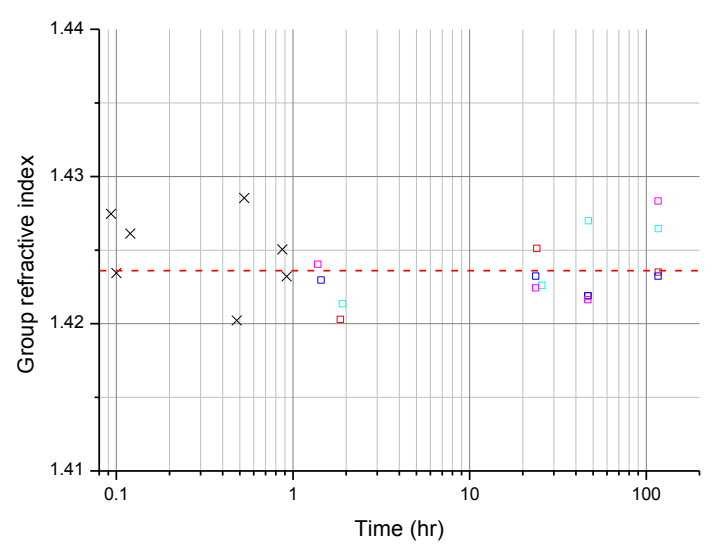

Fig. 4 Time and intersample variation of the measured refractive index for RTT specimens stored in normal saline solution (NSS). Abscissa: time of sample storage in NSS. The squares of the same color correspond to the same RTT fascicle specimen. The crosses correspond to different specimens. The red dash line shows the average value of $n_{g}$ over all experimental points on this graph.

An average value of $n_{g}$ for RTT fascicles in the native state was found to be $1.423 \pm 0.003$ (mean $\pm \mathrm{SD}$; 12 samples). This value is shown by the black triangle in Fig. 5. An average value of $k_{s d r y}$ was $0.323 \pm 0.003$ (mean \pm one-half of the range; 3 samples), and, consequently, the average volume fraction of water for fascicles in the native state can be estimated as $0.677 \pm 0.003$ [see (5)]. Taking $0.677 \pm 0.003$ for $C_{w}$ and 1.34 for the specific gravity $\rho_{d r y}$ of the dry tissue [31], we may estimate the average weight water content for the samples in the native state as $61 \pm 0.3 \mathrm{wt} \%$.

In Fig. 6, the measured values of $n_{g}$ for RTT are plotted against the volume water fraction $C_{w}, C_{w}$ being calculated by (5) at $k_{s d r y}=0.323$. Kim et al. [7] reported the measured values of the group refractive index as a function of a hydration parameter $H$ for bovine cornea at $\lambda_{0}=819.9 \mathrm{~nm}$. The hydration $H$ of tissue is defined as the ratio of the weight of water to the dry weight. Having calculated $C_{w}$ from $H$ as

$$
C_{w}=\frac{H \rho_{d r y}}{1+H \rho_{d r y}}
$$

at $\rho_{d r y}=1.34$, we transferred the experimental data for cornea [7] to Fig. 6 for comparison. It can be seen from the figure that the data for tendon and cornea fit rather well. 


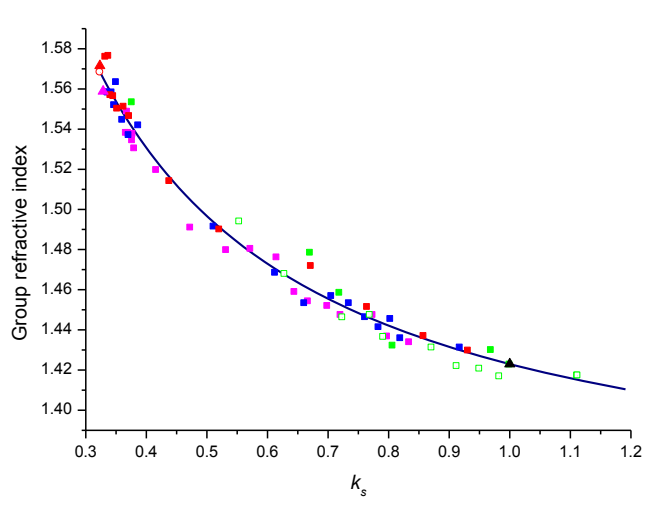

Fig. 5 Group refractive index of RTT fascicles vs volume factor $k_{s}$. The blue squares (a) show experimental points $\left(k_{s}, n_{g}\right)$ for sample 1 (air-dried at room temperature). The red symbols $(\boldsymbol{\square}, \boldsymbol{\Delta}, \circ)$ represent the data for sample 2: the squares ( $\square$ ) are the points obtained during air-drying at room temperature; the open circle $(0)$ and solid triangle $(\Delta)$ show the points obtained on air-drying at $50^{\circ} \mathrm{C}$ and $105^{\circ} \mathrm{C}$, respectively. Purple symbols $(\boldsymbol{\square}, \boldsymbol{\Delta})$ show the data for sample 3: the squares ( $\square$ ) are for air-drying at room temperature, and the triangle $(\Delta)$ is for air-drying at $105^{\circ} \mathrm{C}$. The green symbols $(\square, \square)$ are for sample 4 : the solid squares $(\square)$ are for the stage of air-drying, and open squares $(\square)$ are for the stage of rehydration $(80 \mathrm{~min})$. The black triangle $(\boldsymbol{\Delta})$ shows the average value of $n_{g}$ of RTT fascicles in the native state (over 12 specimens).

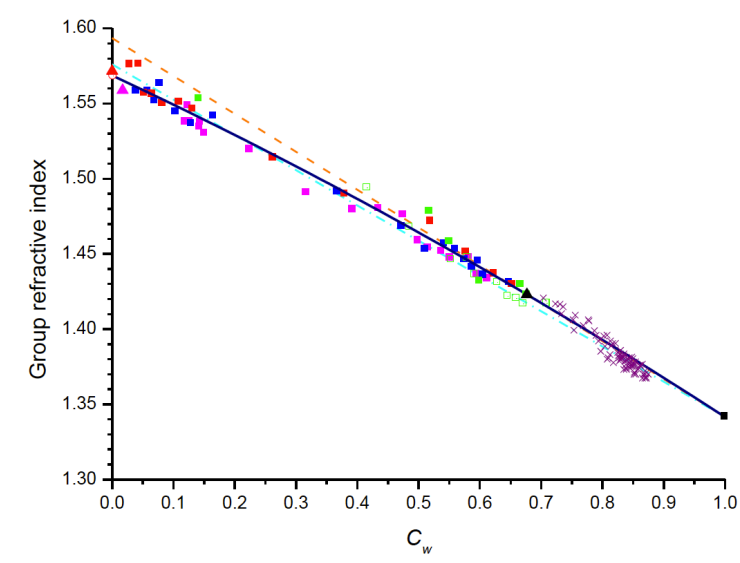

Fig. 6 Group refractive index of RTT fascicles $v s$ volume water content $C_{w}$. The symbols are the same as in Figure 5. The crosses $(\times)$ represent the experimental points for bovine cornea [7] $\left(\lambda_{0}=819.9 \mathrm{~nm}\right)$. The solid line $(-)$ and cyan dash-dot $\left(--_{-}\right)$line represent respectively the approximating quadratic (Eq.9) and linear (Eq.10 with $\left.n_{d r y}=1.576\right)$ polynomials to the data for RTT. The orange dash line $(---)$ is the straight line (Eq.10 with $\left.n_{d r y}=1.594\right)$ passing through the points $\left(C_{w}, n_{g}\right)=(1,1.3416)$ (water) and $(0.677,1.423)$ (the average for the native state of RTT).

The experimental data for RTT are well approximated by the quadratic polynomial

$$
n_{g}=1.5713-0.1969 C_{w}-0.0328 C_{w}^{2}
$$

(Fig. 6). The coefficients of this expression were found by the least squares method under the additional conditions that $n_{g}\left(C_{w}\right)$ must be equal to the group refractive index of water $n_{w}=1.3416$ at $C_{w}=1$ and to the reliable estimate $n_{g}=1.423$ at $C_{w}=0.677$ (native state). At $C_{w}=0$, approximation (9) gives $n_{\mathrm{g}}=1.5713$, which is close to the measured values of the group refractive index for the dry state, $n_{d r y}$. We estimated the accuracy of this and subsequent approximations using the parameter $\sigma_{f i t}=\sqrt{r_{s s} / n}$, where $r_{s s}$ is the residual sum of squares and $n$ is the number of experimental points. For approximation (9), $\sigma_{f i t} \approx 5 \cdot 4 \cdot 10^{-3}$. When approximating the experimental data by a linear function

$$
n_{g}=\left(1-C_{w}\right) n_{d r y}+n_{w} C_{w}
$$

which corresponds to the Gladstone-Dale law [23, 31, 32], with given $n_{w}=1.3416, \sigma_{\text {fit }}$ is minimum (about $6.7 \cdot 10^{-3}$ ) when $n_{d r y}=1.576$ (Fig. 6, cyan line). This approximation gives a significantly underestimated value of $n_{\mathrm{g}}$ (1.417) for the native state. The approximation of the RTT data by the straight line passing through the points $\left(C_{w}, n_{g}\right)=(1,1.3416)$ (water) and $(0.677,1.423)$ (native state), the red line in Fig. 6, ensures a rather good accuracy for the range $0.52 \leq C_{w} \leq 1 \quad\left(\sigma_{f i t} \approx 4 \cdot 10^{-3}\right)$ but gives a significantly overestimated value for $n_{d r y}$ (1.594). The significant deviation of the actual $n_{g}\left(C_{w}\right)$ curve from this approximating line in the region $C_{w} \leq 0.52$ may be attributed to a change of the mode of hydration. In the literature, two modes of collagenous tissue hydration are distinguished [33-36]. For relatively high values of $H$ ( $H>H_{c}$, where $H_{c}$ is a critical value, the so-called fibrillar saturation point [35]), changes in the total water content in the tissue lead to changes of the water content in extrafibrillar space while the water content in collagen fibrils remains unchanged [7, 33-36]. In the alternative mode $\left(H<H_{c}\right)$, changes in the total water content are accompanied by changes in water content both in collagen fibrils and extrafibrillar space [33-36]. For RTT, $H_{c} \approx 0.82[32,35]$, which corresponds to $C_{w} \approx 0.52$.

Fig. 7 shows the dependence of the phase refractive index $n_{p}$ of RTT on $C_{w}$. The phase refractive index was calculated using approximating function (9) and Eq. (6) on the assumption that

$$
\left.\frac{d n_{p}}{d \lambda}\right|_{\lambda=\lambda_{0}}=\left.\left(1-C_{w}\right) \frac{d n_{d r y}}{d \lambda}\right|_{\lambda=\lambda_{0}}+\left.C_{w} \frac{d n_{w}}{d \lambda}\right|_{\lambda=\lambda_{0}} .
$$


The values of $d n_{d r y} / d \lambda$ and $d n_{w} / d \lambda$ were calculated from the dispersion data for gelatin [37] and water [29], respectively. We did not find in the literature any reliable experimental data on the wavelength dependence of the phase refractive index of dry collagen in the spectral range under consideration. Gelatin is known to be produced by partial hydrolysis of animal collagen and to have a very similar chemical structure to collagen. For this reason, we used the available data for dry gelatin. For the phase refractive index of gelatin we used the dispersion formula $n=1.53+1788 / \lambda_{n m}^{2}+5.73 \cdot 10^{8} / \lambda_{n m}^{4}$, where $\lambda_{n m}$ is the numerical value of the wavelength $\lambda$ in nanometers [37].

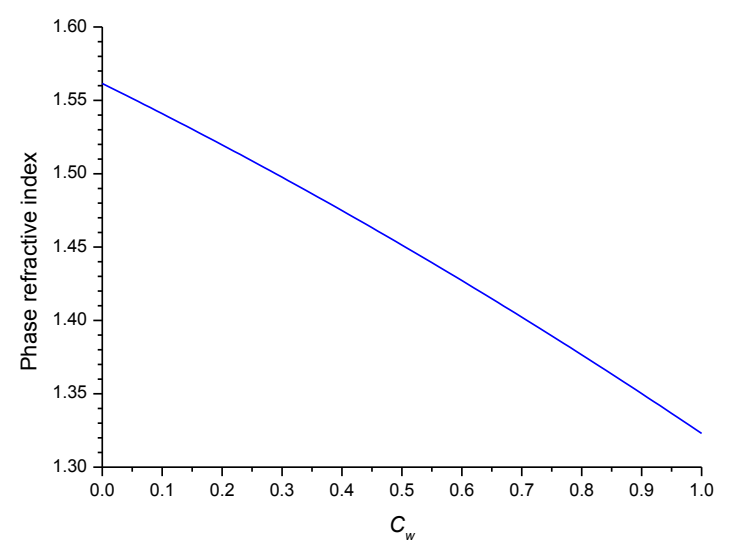

Fig. 7 Tentative estimate of the average phase refractive index of RTT fascicles as a function of volume water content.

\section{Conclusion}

The average group refractive index of RTT fascicles has been measured as a function of water content using OCT. For the native state, this refractive index has been found to be $1.423 \pm 0.003$ at a wavelength of $930 \mathrm{~nm}$. In the range $0.52 \leq C_{w} \leq 1$, the average group refractive index of RTT fascicles has been found to change almost linearly with $C_{w}$. Over the entire range of $C_{w}$ values, $0 \leq C_{w} \leq 1$, the experimental data are well fitted by the quadratic polynomial (9).

\section{Disclosures}

The authors declare that there are no conflicts of interest related to this article.

\section{Acknowledgements}

The study was supported by the Russian Ministry of Education and Science, Project 3.1586.2017/4.6. 